\title{
Correction to: No Stones Left Unturned
}

\section{Correction to: G. Berci, F. L. Greene, No Stones Left Unturned, https://doi.org/10.1007/978-3-030-76845-4}

The below listed changes have been made in the corrected version of the book.

1. The frontispiece on the title page has been placed below the book title.

2. Affiliation for Dr. Bruce Gewertz has been updated in the front matter.

3. Chapter author's names had been missed out in chapters 14 and 17.

Dr. J. Andrew Hamlin has been included as the author for chapter 14 and Professor Alfred Cuschieri has been included as the author for chapter 17.

4. On page 62, Chapter 8: The photographs of the early surgeons have been positioned in portrait position.

5. On page 80, Chapter 13: The photographs of the laparoscopic surgeons have been positioned in portrait position.

The updated online versions of the chapters can be found at https://doi.org/10.1007/978-3-030-76845-4

https://doi.org/10.1007/978-3-030-76845-4_8

https://doi.org/10.1007/978-3-030-76845-4_13

https://doi.org/10.1007/978-3-030-76845-4_14

https://doi.org/10.1007/978-3-030-76845-4_17 